\title{
Fetal Arrhythmias and Related Fetal and Neonatal Outcomes
}

\author{
Fatemeh Abbasalizadeh $^{1}$, Shamsi Abbasalizadeh ${ }^{1}$, Shamsi Ghaffari ${ }^{2}$, Rabee Hesami ${ }^{3}$, Leyla Hesmai ${ }^{*}$
}

\begin{abstract}
Objectives: Fetal cardiac arrhythmia has good prognosis. However, some can lead to hydrops fetalis and perinatal mortality. There are not sufficient studies on the prevalence and complications of fetal arrhythmias in Iran, thus, making parents anxious. Therefore, we performed this study to determine its proper management and to prevent its complications during pregnancy. Then we can help parents by giving them sufficient information about their fetal problem.

Materials and Methods: This descriptive-analytic study included 81 pregnant women with fetal arrhythmia detected in routine monitoring. For cases, we performed fetal echocardiography. Data was analyzed by SPSS $20(P<0.05)$.

Results: This study assessed the fetal period until 28 days after labor. Fetal arrhythmia was confirmed in 37 cases through echocardiography. The most prevalent arrhythmia was extrasystole with 27 cases (72.97\%). Two cases with congenital heart disease experienced heart failure and hydrops fetalis and died in neonatal period and 25 cases survived. Eight cases had bradycardia including 5 with sinus bradycardia and 3 with atrioventricular block. Seven cases had congenital heart problems and 4 of them led to heart failure. Perinatal mortality was seen in six cases. Tachycardia was seen in two cases (5.4\%) with no heart failure or hydrops fetalis. One case died of preterm delivery.

Conclusion: Congenital heart disease is considered as an important risk, affecting fetal and neonatal outcome in fetuses with arrhythmias. As pregnancy advances, the frequency of arrhythmias decreased and converted to sinus rhythm.

Keywords: Arrhythmia, Echocardiography, Fetal monitoring, Hydrops fetalis, Perinatal mortality
\end{abstract}

\section{Introduction}

Studies on fetal cardiac arrhythmia have been increasing during the recent three decades. These arrhythmias are diagnosed during prenatal care through routine fetal cardiac monitoring or routine ultrasound tests carried out during pregnancy. It is confirmed by M-Mod and two-dimensional Doppler echocardiography (1-3). Although most types of the arrhythmias have good prognosis but they can cause problems like hydrops fetalis, congenital heart failure and fetal and neonatal mortality in $1 \%$ to $2 \%$ of cases $(2,4)$.

Fetal arrhythmia is an irregularity in heart rhythm or rate. It can be classified into regular and irregular arrhythmias based on its rhythm and into tachycardia and bradycardia based on its rate. They are detected in about $1 \%$ of pregnancies (3-5). The most common type of irregularity in rhythm is extrasystole arrhythmia resulting from cardiac ectopic beat (6).

Tachycardia and bradycardia are two other types of fetal arrhythmia. Tachycardia in which the heart rate exceeds $160 \mathrm{bpm}$, is divided to sinus tachycardia, supraventricular tachycardia (SVT), atrial flutter, atrial fibrillation and others (6). Most cases of tachyarrhythmia occur during the third trimester of pregnancy $(7,8)$. Bradycardia in which the heart rate is below $110 \mathrm{bpm}$, is divided to atrial-ven- tricular block (AV-block), sinus bradycardia and long QT syndrome. Most of these disorders are associated with structural disorders of the heart or maternal connective tissue disease (6). Isolated complete heart block (CHB) in neonates is rare $(1 / 15000$ to $1 / 20000)$ (9). However, the risk increases to $2 \%$ of pregnancies in women with anti-Ro and anti-La autoantibodies without considering maternal symptoms $(10,11)$. Importantly, the risk of CHB recurrence increases by up to 10 -fold in subsequent pregnancies $(12,13)$.

There are not sufficient studies on the prevalence and complications of fetal arrhythmias in Iran and fetal arrhythmia makes parents anxious. Therefore, we performed this study to investigate the outcome of fetal arrhythmia during pregnancy and neonatal periods in order to determine its proper management and to prevent its complications during pregnancy. Then we can assure parents by giving them sufficient information about their fetal problem.

\section{Materials and Methods}

This study assessed 81 pregnant women who referred to our high-risk pregnancy clinic or to neonatal cardiac specialists because of suspected fetal arrhythmia during routine prenatal care, by auscultation of the fetal heart or

Received 15 February 2015, Accepted 14 August 2015, Available online 5 September 2015

${ }^{1}$ Women's Reproductive Health Research Center, Tabriz University of Medical Sciences, Tabriz, Iran. ${ }^{2}$ Cardiovascular Research Center, Tabriz University of Medical Sciences, Tabriz, Iran. ${ }^{3}$ Urmia University of Medical Sciences, Urmia, Iran.

*Corresponding author: Leyla Hesmai, Women's Reproductive Health Research Center, Tabriz University of Medical Sciences, Tabriz, Iran. Tel: +98 4135541221, Email: sh.hesami90@gmail.com 
by ultrasound. It was performed from October 2012 to October 2014. Among the cases, 27 were inpatients and 54 cases were outpatients, which were referred to pediatric cardiology clinics by obstetricians. To confirm and investigate fetal arrhythmia, fetal echocardiography was conducted on all 81 cases. Those who needed medical intervention received drugs (digoxin, dexamethasone or salbutamol based on the fetal problems) and their response to treatment was studied during pregnancy and neonatal periods. Echocardiographic findings in terms of cardiac anomalies, signs of fetal heart failure and hydrops fetalis were recorded. In addition, fetal and neonatal mortalities (after 28 days from delivery) were investigated. Cases with confirmed fetal arrhythmia were subjected to echocardiography on weeks 34 and 38 of pregnancy and after delivery up to the end of neonatal period and results were recorded in questionnaire. Among the referred cases, those mothers whose fetal arrhythmia was because of maternal fever, labour pain, maternal anemia or chorioamnionitis were excluded.

Collected data was analyzed by SPSS 20 . Chi-square test was used; Fischer test was used in lower number of case. $P<0.05$ was significant. Kappa coefficient was used to determine agreement percentage.

\section{Results}

Eighty-one pregnant women with fetal arrhythmia were enrolled in the study. The average maternal age was $28.13 \pm 5.73$. The average gestational age when fetal arrhythmia was diagnosed, was $29.43 \pm 5.73$ weeks. The average gestational age in delivery was $37.79 \pm 2.5$. Newborn babies had an average weight of $3079.25 \pm 583.92$ grams. Among 81 cases, one woman (1.2\%) had a history of cardiac anomaly.

Among 81 pregnant women referring because of arrhythmia, arrhythmia was confirmed in 37 cases $(45.67 \%)$ by echocardiography. Table 1 shows the frequency of different causes of arrhythmias when referred to pediatric cardiologist by obstetricians.

From 37 cases with fetal arrhythmia confirmed by echocardiography, the most frequent arrhythmia was extrasystole seen in 27 cases $(72.97 \%)$. Other types were sinus bradycardia in five cases (13.51\%), AV-block in 3 cases (8.1\%) and tachycardia in 2 cases (5.4\%).

Among cases whom arrhythmia was confirmed by echocardiography, 12 cases needed medical therapy during pregnancy, 8 of which $(66.7 \%)$ responded to the treatment, while 4 cases $(33.33 \%)$ did not respond, which in- cluded one extrasystole, one tachycardia (SVT type) and 2 cases were $\mathrm{CHB}$ who required pace-maker after birth.

By ultrasound examination, six cases $(7.4 \%)$ were diagnosed as fetal anomaly, five of which had hydrops fetalis. However, ultrasound was normal in 75 cases (92.6\%). Echocardiographic findings of 15 cases (18.51\%) out of 81 cases were abnormal.

About the fetal and neonatal outcomes, the frequency of perinatal mortality of different fetal arrhythmias was nine (24.32\%). Among the dead neonates, 8 (88.88\%) had heart anomaly of which two had extrasystole (one case had hypoplastic left heart problem and the other case had ventricular septal defect plus pericardial effusion and hydrops fetalis), three had sinus bradycardia and all of them had heart anomaly. One had AV-channel defect, one had hypoplastic left heart syndrome with hydrops fetalis and the last case had atrial septal defect with cardiomegaly and heart failure. Two of the dead neonates had AV-block arrhythmia and both had pleural effusion and heart failure. One of the dead neonates had sinus tachycardia that died at the 30th week of pregnancy due to preterm birth and respiratory distress with no heart anomaly.

About the fetal and neonatal complications in extrasystole cases: Among 27 cases with extrasystole, arrhythmia resolved in 24 cases $(88.88 \%)$. Two cases $(7.4 \%)$ with extrasystole arrhythmia had hydrops fetalis and heart failure. There was not any Intrauterine Fetal Death (IUFD) in the extrasystole cases. Neonatal death was seen in two cases (7.4\%), both of which had heart anomaly and heart failure. Among cases (22.2\%) with extrasystole, cardiac anomaly was seen in six cases by echocardiography. 25 (92.59\%) of extrasystole cases survived.

About the fetal and neonatal complications in cases with sinus bradycardia: Among five sinus bradycardia cases, the problem resolved in one case (20\%). Two cases (40\%) had heart failure, one of which was associated with hydrops fetalis. One case $(20 \%)$ had hydrops fetalis. One case died during fetal period and perinatal mortality was seen in four cases (80\%); all of them had heart anomaly. Only one case (20\%) survived.

About the fetal and neonatal complications in cases with tachycardia: As mentioned there were only 2 cases with tachycardia; one resolved during pregnancy and one had preterm birth and died because of it. Thus, it was not possible to evaluate the fetal and neonatal outcome of this arrhythmia.

About the fetal and neonatal complications in cases with AV- block arrhythmia: There were three cases with this

Table 1. Fetal Arrhythmia Detected in Routine Examination Versus Verified Heart Arrhythmia by Echocardiography

\begin{tabular}{lcc}
\hline \multirow{2}{*}{ Verified Heart Arrhythmia by Echocardiography, $\mathbf{n = 3 7}$} & \multicolumn{2}{c}{ Fetal Arrhythmia Detected In Routine Examination, $\mathbf{n}=\mathbf{8 1}$} \\
\cline { 2 - 3 } Extrasystole $(\mathrm{n}=27)$ & Extrasystole, $\mathbf{n = 5 8}$ & Bradycardia, $\mathbf{n = 1 2}$ \\
Tachycardia $(\mathrm{n}=2)$ & 27 & - \\
Bradycardia $(\mathrm{n}=5)$ & - & - \\
Atrioventricular block $(\mathrm{n}=3)$ & - & 5 \\
Normal sinus rhythm $(\mathrm{n}=44)$ & - & 3 \\
\hline
\end{tabular}


problem; hydrops fetalis and heart failure were seen in two cases $(66.66 \%)$. Neonatal mortality was seen in two cases (66.66\%); both of them had cardiac anomaly and one case with complete cardiac block was candidate for embedding a pacemaker but died before the operation. The other case had second degree heart block. The survived case had CHB without cardiac anomaly. Her mother suffered from lupus. After birth, a pacemaker was embedded and the neonate is being treated with dexamethasone and salbutamol since the fetal period. Table 2 summarizes the obtained results.

As pregnancy progresses, the vast majority of fetal cardiac arrhythmias turn to sinusoidal rhythm; thus in this study the number of arrhythmia cases reduced to $12(14.81 \%)$ with 69 cases $(85.18 \%)$ having normal sinus rhythm. Figures 1 and 2 show the frequency of arrhythmias at the time of diagnosis, late pregnancy and neonatal period.

\section{Discussion}

In Vergani et al study, the prevalence of extrasystole arrhythmia was 88 cases $(77 \%) ; 88 \%$ of which turned to normal sinusoidal rhythm. None of the cases had heart anomaly and heart failure. However, no mortality was seen during fetal and neonatal periods. In this study, 100\% of cases survived (5). In our study the prevalence of extrasystole was the same as Vergani et al study; however the number of survived cases was higher and no mortality and heart failure was seen in that study which could be because that none of their fetuses had cardiac anomaly. In Rasiah et al study, sinusoidal bradycardia was seen 15

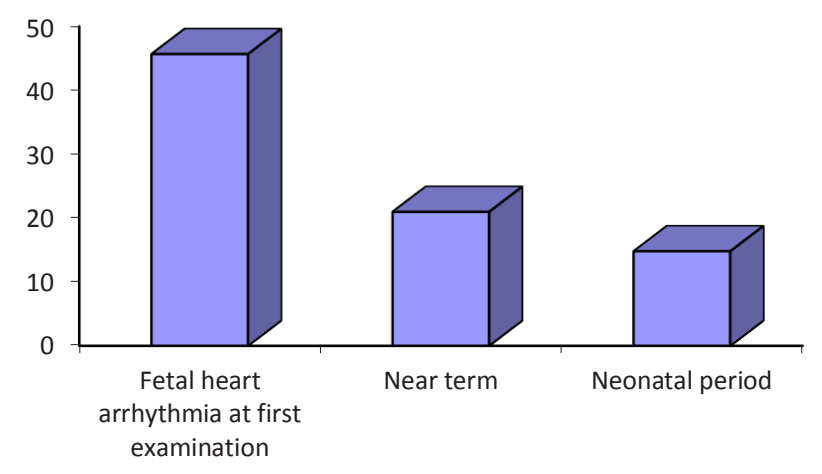

Figure 1. Frequency of fetal arrhythmias in progress of gestational age and neonatal period. cases $(8.24 \%) ; 10$ of them $(66.66 \%)$ had congenital heart problem. Survival rate in cases with no heart problem and in cases with heart problem was $73 \%$ and $20 \%$, respectively (2). Our study similar to Rasiah et al study showed the important role of contemporary fetal cardiac anomalies in causing fetal and neonatal complications in sinus bradycardia.

In Vergani et al study, AV-block was observed in four cases. In one case (25\%) AV-block turned to sinus rhythm. Three cases had heart failure; two of them died (5). The prevalence of AV-block in our study was nearly similar to Vergani et al study, but with more serious complications and arrhythmia related mortality increased in the presence of congenital heart disease and heart failure.

In Vergani et al study, tachycardia was observed in 17 cases (14.91\%), 14 of which $(82.35 \%)$ were treated. However, three SVT cases survived up to one year. They were treating by drug during this period. Heart failure, cardiac anomaly and fetal and neonatal mortality were seen in 5 (29.41\%), 2 (11.76\%), and 1 (5.88\%) cases, respectively (5).

In our study, we had only 2 cases with tachycardia and among them there was no heart failure, hydrops fetalis and turning to sinus rhythm. The reason may be the smaller size of our study. Maybe the emergency termination of pregnancies complicated by this arrhythmia in our study was the reason, as there was no sufficient time for studying this arrhythmia. Mortality rate was higher in our study. The reason could be preterm birth and neonatal respiratory distress syndrome. Therefore, fetal arrhythmias

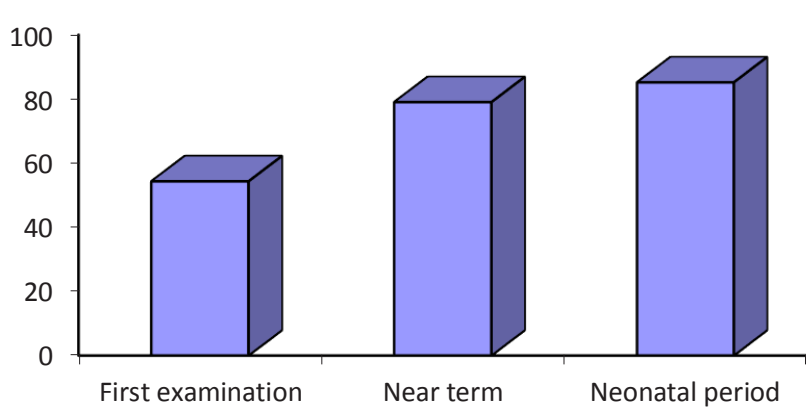

Figure 2. Frequency of Normal Sinus Rhythm in 81 Suspicious Fetal Arrhythmia Cases in Progressive Gestational Age and Neonatal Period.

Table 2. Outcome of Fetal and Neonatal Arrhythmias

\begin{tabular}{lccccc}
\hline \multirow{2}{*}{ Outcome } & \multicolumn{5}{c}{ Arrhythmia } \\
\cline { 2 - 5 } & Extrasystole & Sinus Bradycardia & Tachycardia & AV-Block & Total \\
\hline Normal sinus rhythm & $\mathrm{n}=24(88.88 \%)$ & $\mathrm{n}=1(20 \%)$ & $0 \%$ & $0 \%$ & 25 \\
Hydrops fetalis & $\mathrm{n}=2(7.4 \%)$ & $\mathrm{n}=1(20 \%)$ & $0 \%$ & $\mathrm{n}=2(66.66 \%)$ & 5 \\
IUFD & $0 \%$ & $\mathrm{n}=1(20 \%)$ & $0 \%$ & $0 \%$ & 1 \\
Neonatal death & $\mathrm{n}=2(7.4 \%)$ & $\mathrm{n}=3(60 \%)$ & $\mathrm{n}=1(50 \%)$ & $\mathrm{n}=2(66.66 \%)$ & 8 \\
Abnormal findings in echocardiography & $\mathrm{n}=6(22.22 \%)$ & $\mathrm{n}=5(100 \%)$ & $0 \%$ & $\mathrm{n}=2(66.66 \%)$ & 13 \\
Survivals & $\mathrm{n}=25(92.59 \%)$ & $\mathrm{n}=1(20 \%)$ & $\mathrm{n}=1(50 \%)$ & $\mathrm{n}=1(33.33 \%)$ & 28 \\
Heart failure & $\mathrm{n}=2(4.7 \%)$ & $\mathrm{n}=2(60 \%)$ & $0 \%$ & $\mathrm{n}=2(66.66 \%)$ & 6 \\
Total & 27 & 5 & 2 & 3 & 37 \\
\hline
\end{tabular}

Abbreviation: IUFD, Intrauterine Fetal Death. 
could not be considered as the cause of mortality with high reliability. And it is better not to terminate these cases preterm only because of arrhythmia.

\section{Conclusion}

In our study, almost $50 \%$ of fetal arrhythmias discovered by routine fetal monitoring were confirmed by echocardiography; the vast majority of which had reached term pregnancy. Congenital heart diseases are considered as important risk factors of all arrhythmias resulting in fetal and neonatal complications and perinatal mortality. As pregnancy progresses, correction to sinusoidal rhythm increases with extrasystole which is the most correctable arrhythmia.

It can be argued, therefore, that pregnant mothers with fetal cardiac arrhythmia of extrasystole type can be assured that the vast majority of cases will be corrected during fetal period and this problem has good prognosis. However, cases with bradycardia or with heart anomaly should be advised to refer to pediatric cardiologist for sufficient investigation and to deliver their baby at level 3 hospitals.

\section{Ethical Issues}

The Ethics Committee of Tabriz University of Medical Sciences approved this study.

\section{Conflict of Interests}

The authors declare no conflict of interests.

\section{Financial Support}

Not applicable.

\section{Acknowledgments}

None to be declared.

\section{References}

1. Maeno Y, Hirose A, Kanbe T, Hori D. Fetal arrhythmia: prenatal diagnosis and perinatal management. J Obstet Gynaecol Res. 2009;35(4):623-629. doi:10.1111/j.14470756.2009.01080.x.

2. Rasiah SV, Ewer AK, Miller P, Kilby MD. Prenatal diagnosis, management and outcome of fetal dysrhythmia: a tertiary fetal medicine centre experience over an eightyear period. Fetal Diag Ther. 2011;30(2):122-127. doi: $10.1159 / 000325464$

3. Namouz-Haddad S, Koren G. Fetal Pharmacotherapy 2:
Fetal Arrhythmia. J Obstet Gynaecol Can. 2013;35(11):10231027.

4. Hahurij ND, Blom NA, Lopriore E, et al. Perinatal management and long-term cardiac outcome in fetal arrhythmia. Early Hum Dev. 2011;87(2):83-87. doi:10.1016/j.earlhumdev.2010.11.001.

5. Vergani P, Mariani E, Ciriello E, et al. Fetal arrhythmias: natural history and management. Ultrasound Med Biol. 2005;31(1):1-6. doi:10.1016/j.ultrasmedbio.2004.10.001.

6. James DK, Steer PJ, Weiner CP, Gonik B. High risk pregnancy: management options-expert consult. Elsevier Health Sciences; 2010.

7. Krapp M, Kohl T, Simpson JM, Sharland GK, Katalinic A, Gembruch U. Review of diagnosis, treatment, and outcome of fetal atrial flutter compared with supraventricular tachycardia. Heart. 2003;89(8):913-917. doi:10.1136/ heart.89.8.913.

8. Jaeggi ET, Carvalho JS, De Groot E, et al. Comparison of transplacental treatment of fetal supraventricular tachyarrhythmias with digoxin, flecainide and sotalol results of a nonrandomized multicenter study. Circulation. 2011;124(16):1747-54. doi:10.1161/ CIRCULATIONAHA.111.026120.

9. Eliasson H, Sonesson SE, Sharland G, et al. Isolated atrioventricular block in the fetus a retrospective, multinational, multicenter study of 175 patients. Circulation. 2011;124(18):1919-1926. doi:10.1161/ CIRCULATIONAHA.111.041970.

10. Jaeggi ET, Fouron JC, Silverman ED, Ryan G, Smallhorn J, Hornberger LK. Transplacental fetal treatment improves the outcome of prenatally diagnosed complete atrioventricular block without structural heart disease. Circulation. 2004;110(12):1542-1548. doi:10.1161/01. CIR.0000142046.58632.3A.

11. Izmirly PM, Buyon JP, Saxena A. Neonatal lupus: advances in understanding pathogenesis and identifying treatments of cardiac disease. Curr Opin Rheumatol. 2012;24(5):466. doi:10.1097/BOR.0b013e328356226b.

12. Friedman DM, Llanos C, Izmirly PM, et al. Evaluation of fetuses in a study of intravenous immunoglobulin as preventive therapy for congenital heart block: Results of a multicenter, prospective, open-label clinical trial. Arthritis Rheumatism. 2010;62(4):1138-1146. doi:10.1002/art.27308.

13. Kaaja $R$, Julkunen $H$. Prevention of recurrence of congenital heart block with intravenous immunoglobulin and corticosteroid therapy: comment on the editorial by Buyon et al. Arthritis Rheumatism. 2003;48(1):280-281. doi:10.1002/art.10716.

Copyright (C) 2016 The Author(s); This is an open-access article distributed under the terms of the Creative Commons Attribution License (http://creativecommons.org/licenses/by/4.0), which permits unrestricted use, distribution, and reproduction in any medium, provided the original work is properly cited. 